\title{
HYPERCONTRACTIVITY ESTIMATES FOR NONSELFADJOINT DIFFUSION SEMIGROUPS
}

\author{
ROSS PINSKY \\ (Communicated by John B. Conway)
}

\begin{abstract}
We use Gross' results on logarithmic Sobolev inequalities and hypercontractivity to show that the hypercontractivity of a positive recurrent diffusion semigroup depends essentially only on its invariant probability measure and a given diffusion matrix and its diffusion matrix. Thus all diffusion semigroups (including the unique selfadjoint one) with a given invariant probability measure and a given diffusion matrix are essentially equally hypercontractive.
\end{abstract}

With the exception of [2], the literature on hypercontractivity estimates seems to deal exclusively with selfadjoint operators. In this note, we show how such estimates extend easily to nonselfadjoint diffusion semigroups when these latter are considered as operators on $L^{p}$ with an appropriate reference measure. More specifically, we will show that the hypercontractivity properties of a positive recurrent diffusion semigroup depend essentially only on the invariant probability measure and the diffusion matrix of the diffusion. All diffusion semigroups with a given invariant probability measure $\mu$ and a given diffusion matrix (including the unique one that is selfadjoint on $L^{2}(d \mu)$ ) are essentially equally hypercontractive on $L^{p}(d \mu)$.

We shall consider the following two classes of diffusion semigroups.

I. Diffusion in a bounded region with oblique reflection at the boundary. $T_{t}$ is the unique diffusion semigroup generated by an extension of $(L, \underline{D})$ where $L=\frac{1}{2} \nabla \cdot a \nabla+b \cdot \nabla$ and $\underline{D}=\left\{u \in C^{2}(\bar{D}): J \cdot \nabla u=0\right.$ on $\left.\partial D\right\}$. We assume that $D \subset R^{d}$ is a bounded domain defined by $\{\psi>0\}$ where $\psi \in C^{2, \alpha}\left(R^{d}\right)$ and $|\nabla \psi| \neq 0$ on $\psi=0$, that is, on $\partial D$, that $a=a(x)$ is a $d \times d$ positive definite matrix for each $x \in R^{d}$ with entries $a_{i j} \in C^{1, \alpha}\left(R^{d}\right)$, that $b$ is a $d$-vector with components, $b_{i} \in C^{\alpha}\left(R^{d}\right)$ and that $J$ is a $d$-vector in $C^{1, \alpha}(\partial D)$ and satisfies $J \cdot n<0$ on $\partial D$, where $n$ is the outward unit normal. The existence and uniqueness of such a diffusion semigroup may be found in [5]. By the compactness of $\partial D$, the positivity of $a$ and the conditions on $J$, we may write $J$ as $J=-a n+T$ where $T$ is a $C^{1, \alpha}$-vector field on the tangent space to $\partial D$. Because the state space is compact and $L$ is uniformly elliptic, a unique invariant probability measure $\mu$ exists for the process (that is, a measure $\mu$ for which $T_{t}^{*} \mu=\mu$ for all $t>0$ ) and possesses a strictly positive density which we will denote by $d \mu / d x=e^{2 Q}$.

Received by the editors October 6, 1987.

1980 Mathematics Subject Classification (1985 Revision). Primary 46E35, 60J60.

Key words and phrases. Hypercontractivity, logarithmic Sobolev inequalities, nonselfadjoint elliptic operator, diffusion semigroup.

This work was supported by the fund for the promotion of research at the Technion. 
II. Diffusion on all of $R^{d}$. $T_{t}$ is the unique diffusion semigroup generated by an extension of $(L, \underline{D})$ where $L=\frac{1}{2} \nabla \cdot a \nabla+b \cdot \nabla$ and $\underline{D}=\left\{u \in C_{0}\left(R^{d}\right) \cap C^{2}\left(R^{d}\right)\right\}$, where $a=a(x)$ is a positive definite $d \times d$ matrix for each $x \in R^{d}$ with entries $a_{i j} \in C^{1}\left(R^{d}\right)$ and $b$ is a $d$-vector with components $b_{i} \in C\left(R^{d}\right)$.

Before stating our theorem, we present a compilation of facts concerning when a diffusion process will possess a given invariant measure and when its semigroup is selfadjoint with respect to some reference measure. We first consider the case of diffusions with reflection. The diffusion semigroup will be selfadjoint on $L^{2}$ with respect to some reference measure if and only if $b$ is of the form $a \nabla Q$ for some function $Q$ and $J=-a n$, that is $T \equiv 0$. In this case the invariant probability measure $\mu$ will possess the density $d \mu / d x=e^{2 Q}$. (Since the diffusion depends on $\nabla Q$ but not on $Q$ we may assume without loss of generality that $Q$ has been picked so that $e^{2 Q}$ integrates to one.) The diffusion semigroup will be selfadjoint on $L^{2}(D, d \mu)$ and its generator $L$ will possess the quadratic form $Q_{L}(f, g)=\int_{D}(\nabla f a \nabla g) e^{2 Q} d x$. In dimension $d=1$, the above scenario is the only possible one; hence all one dimensional diffusion semigroups are selfadjoint on $L^{2}(D, d \mu)$. However, in dimension $d \geq 2$, there are many other nonselfadjoint diffusion semigroups with the same invariant measure $\mu$ (see [4] for a classification of such diffusions).

The following analytic criterion for determining when a particular probability measure is invariant for a given reflecting diffusion is useful and will be used in the sequel.

PROPOSITION 1. Consider a reflected diffusion on $D$ generated by $L=$ $\frac{1}{2} \nabla \cdot a \nabla+b \cdot \nabla$ with reflection vector $J=-a n+T$. Assume $a, b, J$ and $D$ satisfy the conditions given above for reflected diffusions. Let $\mu$ be a probability measure on $D$ with density $d \mu / d x=e^{2 Q}$, where $Q \in C^{1, \alpha}(\bar{D})$ and write $b$ in the form $b=a \nabla Q+(b-a \nabla Q) \equiv a \nabla Q+\tilde{b}$. If $\mu$ is invariant for the diffusion process, then

$$
\int_{D} \tilde{b} \nabla q e^{2 Q} d x+\frac{1}{2} \int_{\partial D}(\nabla q \cdot T) e^{2 Q} d \sigma=0 \quad \text { for all } q \in W^{1,2}(D) .
$$

PROOF. It is well known that $\mu$ is invariant if and only if $\int_{D} L q d \mu=0$ for all $q$ in the domain of $L$. Take $q \in \underline{D}$ and integrate by parts. Then take a limit to get (1.1) for $q \in W^{1,2}(D)$.

In the case of a diffusion on all of $R^{d}$, we need the following assumption.

AsSUMPTION A. (i) The process possesses an invariant probability measure $\mu$ with $d \mu / d x=e^{2 Q}$ and drift $b$ of the form $b=a \nabla Q+\tilde{b}$ where $\tilde{b} \in L^{1}\left(R^{d}, d \mu\right)$;

(ii) $\liminf \operatorname{in}_{m \rightarrow \infty} \mid \int_{|x|=m}$ ane $e^{2 Q} d \sigma \mid=0$, where $n$ is the outward unit normal at $|x|=m$.

Note that since $e^{2 Q}$ is integrable, (ii) is always satisfied if $a$ is bounded. We have the following proposition.

Proposition 2. Consider a diffusion on $R^{d}$ generated by $L=\frac{1}{2} \nabla \cdot a \nabla+b \cdot \nabla$, where $a_{i j} \in C^{1}\left(R^{d}\right)$ and $b_{i} \in C\left(R^{d}\right)$. Let $\mu$ be a probability measure on $R^{d}$ with density $d \mu / d x=e^{2 Q}$, where $Q \in C^{1}\left(R^{d}\right)$ and write $b$ in the form $b=a \nabla Q+$ $(b-a \nabla Q) \equiv a \nabla Q+\tilde{b}$. Assume that Assumption A holds. If $\mu$ is invariant for the diffusion process, then

$$
\int_{R^{d}} \tilde{b} \nabla q e^{2 Q} d x=0 \quad \text { for all } q \in C_{b}^{2}\left(R^{d}\right) \cap \underline{D}(L)_{p}, \quad \text { for any } p \geq 1 .
$$


(Here and in the sequel, we use $C_{b}^{m}\left(R^{d}\right)$ to denote the space of bounded functions on $R^{d}$ with $m$ continuous bounded derivatives. $\underline{D}(L)_{p}$ denotes the domain of the semigroup when considered as an operator on $L^{p}\left(R^{d}, d \mu\right)$.)

PrOOF. Proceed as in the proof of Proposition 1 and use Assumption A.

For the case of a diffusion on all of $R^{d}$, we will also need the following assumption.

Assumption B. $T_{t}$ leaves $C_{b}^{2}\left(R^{d}\right)$ invariant.

We now present our theorem, but first a word on notation. We will use the generic $\Omega$ to denote either $D$ or $R^{d}$ according to whether we are considering the diffusion on $D$ with reflection or the diffusion on all of $R^{d}$. Also $\underline{P}(\Omega)$ will denote the space of probability measures on $\Omega,\|\cdot\|_{p, \mu}$ will denote the $L^{p}(\Omega, d \mu)$ norm, $\|\cdot\|_{p, q ; \mu}$ will denote the operator norm from $L^{p}(\Omega, d \mu)$ to $L^{q}(\Omega, d \mu)$ and $(\cdot, \cdot)_{\mu}$ will denote the $L^{2}$-inner product with respect to the measure $\mu$.

THEOREM. Let $\mu \in \underline{P}(\Omega)$ with density $d \mu / d x=e^{2 Q}$ where $Q \in C^{1, \alpha}(\bar{D})$ or $C^{1}\left(R^{d}\right)$ according to whether $\Omega=D$ or $\Omega=R^{d}$. Assume there exists an interval $(\alpha, \beta)$ with $1 \leq \alpha<\beta \leq \infty$ and continuous functions $c(\cdot)>0$ and $\gamma(\cdot)$ on $(\alpha, \beta)$ such that

$$
\begin{array}{r}
\int_{\Omega} f^{p} \log |f| d \mu \leq \frac{p-1}{2} c(p) \int_{\Omega}(\nabla f a \nabla f) f^{p-2} d \mu+c(p) \gamma(p)\|f\|_{p, \mu}^{p} \\
+\|f\|_{p, \mu}^{p} \log \|f\|_{p, \mu}, \quad \text { for all } f \in W^{1,2}(\Omega, d \mu) \cap L^{p}(\Omega, d \mu)
\end{array}
$$

(In particular, if (1.3) holds for $p=2$ with constants $c_{0}$ and $\gamma_{0}$, then it holds for all $p$ with $c(p)=c_{0} p / 2(p-1)$ and $\gamma(p)=\gamma_{0}$.) Let $p(t, q)$ be the solution of the initial value problem $c(p) d p / d t=p, p(0, q)=q, t \geq 0$ and let $M(t, q)=\int_{0}^{t} \gamma(p(s, q)) d s$. $p(t, q)$ and $M(t, q)$ are both defined as long as $p(t, q)<\beta$. Consider the two classes of diffusion semigroups defined above and assume that Assumptions $A$ and $B$ hold in the case $\Omega=R^{d}$. Then every such diffusion semigroup $T_{t}$ whose invariant measure is $\mu$ and whose diffusion matrix is a satisfies

$$
\left\|T_{t}\right\|_{q, p(t, q) ; \mu} \leq e^{M(t, q)} .
$$

Conversely, assume there exist continuous $\rho(t, q)$ and $m(t, q)$ on an interval $[0, \varepsilon(q))$, $\varepsilon(q)>0$ with $\rho(0, q)=q$ and $m(0, q)=1$ and such that the right derivatives of $\rho$ and $m$ exist at $t=0$. If the inequality

$$
\left\|T_{t}\right\|_{q, \rho(t, q) ; \mu} \leq m(t, q), \quad 0 \leq t<\varepsilon(\theta), q \in(\alpha, \beta)
$$

holds for one such diffusion semigroup $T_{t}$ with invariant measure $\mu$ and diffusion matrix a, then in fact $\left\|T_{t}\right\|_{q, p(t, q) ; \mu} \leq e^{M(t, q)}$ for every such diffusion semigroup $T_{t}$ with invariant measure $\mu$ and diffusion matrix $a$, where $p(t, q)$ and $M(t, q)$ are as defined above with $c(\cdot)$ and $\gamma(\cdot)$ given by

$$
c(q)=\left.\frac{q}{d \rho(t, q) / d t}\right|_{t=0}
$$

and $\gamma(q)=d m(t, q) /\left.d t\right|_{t=0}$.

PROOF. The proof as well as the formulation of this theorem is based on Gross' paper [1], specifically Theorems 1 and 2. In fact, a reading of these theorems 
and Corollary 2.1 shows that it suffices to establish that for any such diffusion semigroup,

$$
\left(-L f, f^{p-1}\right)_{\mu}=\frac{p-1}{2} \int_{\Omega}(\nabla f a \nabla f) f^{p-2} d \mu .
$$

for all $0 \leq f \in \underline{D}(L)_{p}$, where $\underline{D}(L)_{p}$ is the domain of $L$ when considered on $L_{p}(\Omega, d \mu)$. The first problem with this is that, in the nonselfadjoint case, an explicit operator core cannot generally be given. However, a look at Gross' proof reveals that it suffices to show that (1.4) holds for $0 \leq f \subset \underline{G}$ where $\underline{G} \subset \underline{D}(L)_{p}$ is dense in $L^{p}(\Omega, d \mu)$ and is invariant under $T_{t}$. Now, in the case of a reflected diffusion, Proposition 3, stated and proved below, states that $C^{2, \alpha}(\bar{D})$ is invariant under $T_{t}$. Thus, in this case we will use $\underline{G}=C^{2, \alpha}(\bar{D}) \cap \underline{D}(L)_{p}$. In the case of a diffusion on all of $R^{d}, C_{b}^{2}\left(R^{d}\right)$ is invariant under $T_{t}$ by Assumption B. Thus in this case we will use $\underline{G}=C_{b}^{2}\left(R^{d}\right) \cap \underline{D}(L)_{p}$.

We first consider the case of a reflected diffusion. We will show that

$$
\left(-L f, f^{p-1}\right)_{\mu}=\frac{p-1}{2} \int_{\Omega}(\nabla f a \nabla f) f^{p-2} d \mu, \quad \text { for all } 0 \leq f \in C^{2, \alpha}(\bar{D}) \cap \underline{D}(L)_{p}
$$

Since $f$ is smooth and $f \in \underline{D}(L)_{p}, f$ must satisfy the boundary condition $J \cdot \nabla f \equiv$ $-n a \nabla f+T \cdot \nabla f=0$ on $\partial D$. Integrating by parts, we obtain

$$
\begin{aligned}
\left(-L f, f^{p-1}\right)_{\mu}= & -\int_{D}\left(\frac{1}{2} \nabla \cdot a \nabla f+b \nabla f\right) f^{p-1} e^{2 Q} d x \\
= & \frac{p-1}{2} \int_{D}(\nabla f a \nabla f) f^{p-2} e^{2 Q} d x-\int_{D} \nabla f(b-a \nabla Q) f^{p-1} e^{2 Q} d x \\
& -\frac{1}{2} \int_{\partial D}(n a \nabla f) f^{p-1} e^{2 Q} d \sigma \\
= & \frac{p-1}{2} \int_{D}(\nabla f a \nabla f) f^{p-2} e^{2 Q} d x-\int_{D}(b-a \nabla Q) \nabla\left(\frac{f^{p}}{p}\right) e^{2 Q} d x \\
& -\frac{1}{2} \int_{\partial D} \nabla\left(\frac{f^{p}}{p}\right) \cdot T d \sigma \\
= & \frac{p-1}{2} \int_{D}(\nabla f a \nabla f) f^{p-2} e^{2 Q} d x,
\end{aligned}
$$

where we have used the boundary condition in the third equality and Proposition 1 in the last equality.

We now consider the case of a diffusion in all of $R^{d}$. We will show that

$$
\left(-L f, f^{p-1}\right) \mu=\int_{R^{d}}(\nabla f a \nabla f) f^{p-2} e^{2 Q} d x \quad \text { for all } 0 \leq f \in C_{b}^{2}\left(R^{d}\right) \cap \underline{D}(L)_{p}
$$


Let $B_{m}=\{|x|<m\}$. Then $\left(-L f, f^{p-1}\right)_{\mu}=-\lim _{m \rightarrow \infty} \int_{B_{m}} f^{p-1} L f e^{2 Q} d x$ and integrating by parts gives

$$
\begin{aligned}
-\int_{B_{m}} f^{p-1} L f e^{2 Q} d x= & \int_{B_{m}}(\nabla f a \nabla f) f^{p-2} e^{2 Q} d x-\int_{B_{m}} \nabla f(b-a \nabla Q) f^{p-1} e^{2 Q} d x \\
& -\frac{1}{2} \int_{\partial B_{m}}(n a \nabla f) f^{p-1} e^{2 Q} d \sigma \\
= & \int_{B_{m}}(\nabla f a \nabla f) f^{p-2} e^{2 Q} d x-\int_{B_{m}} \tilde{b} \nabla\left(\frac{f^{p}}{p}\right) e^{2 Q} d x \\
& -\frac{1}{2} \int_{\partial D}(n a \nabla f) f^{p-1} e^{2 Q} d \sigma .
\end{aligned}
$$

By Proposition 2, Assumption $A$ and the fact that $f \in C_{b}^{2}\left(R^{d}\right)$, it follows that $\left(-L f, f^{p-1}\right) \mu=\int_{R^{d}}(\nabla f a \nabla f) f^{p-2} e^{2 Q} d x$.

We now state and prove Proposition 3.

PROPOSITION 3. Let $T_{t}$ be the semigroup of a diffusion with oblique reflection as defined above. Then $T_{t}$ leaves $C_{2, \alpha}(\bar{D})$ invariant.

PROOF. This proposition follows from [3, Theorem 5.3] since $w(t, x) \equiv T_{t} f(x)$ satisfies $w_{t}=L w$ with initial condition $w(0, x)=f(x)$ and boundary condition $J \cdot \nabla w=0$ on $\partial D$, if $f \in \underline{D}(L)_{p}$.

\section{REFERENCES}

1. L. Gross, Logarithmic Sobolev inequalities, Amer. J. Math. 97 (1975), 1061-1083.

2. S. Kusuoka, and D. W. Stroock, Some boundedness properties of certain stationary diffusion semigroups, J. Funct. Anal. 60 (1985), 243-264.

3. O. A. Ladyzenskaja, V. A. Solonnikov and N. N. Ural'ceva, Linear and quasilinear equations of parabolic type, Trans. Math. Monographs, vol. 23, Amer. Math. Soc., Providence, R.I., 1968.

4. R. G. Pinsky, A classification of diffusion processes with boundaries by their invariant measures, Ann. of Probab. 13 (1985), 693-697.

5. D. W. Stroock and S. R. S. Varadhan, Diffusion processes with boundary conditions, Comm. Pure Appl. Math. 24 (1971), 147-225.

Department of Mathematics, Technion-IsRael Institute of Technology, HAIFA 320000, ISRAEL 